\title{
Early results from the SAGE-SMC Spitzer legacy
}

Karl D. Gordon ${ }^{1}$, M. Meixner, R. D. Blum, W. Reach, B. A. Whitney, J. Harris, R. Indebetouw, A. D. Bolatto, J.-P. Bernard, M. Sewilo, B. L. Babler, M. Block, C. Bot, S. Bracker, L. Carlson, E. Churchwell, G. C. Clayton, M. Cohen, C. W. Engelbracht, Y. Fukui, V. Gorjian, S. Hony, J. L. Hora, F. Israel, A. Kawamura, A. K. Leroy, A. Li, S. Madden, A. R. Marble, F. Markwick-Kemper, M. Meade, K. A. Misselt, A. Mizuno, N. Mizuno, E. Muller, J. M. Oliveira, K. Olsen, T. Onishi, R. Paladini, D. Paradis, S. Points, T. Robitaille, D. Rubin, K. M. Sandstrom, S. Sato, H. Shibai, J. D. Simon, L. J. Smith, S. Srinivasan, A. G. G. M. Tielens, U. P. Vijh, S. van Dyk, J. Th. van Loon, K. Volk and D. Zaritsky

${ }^{1}$ Space Telescope Science Institute, 3700 San Martin Drive, Baltimore, MD 21218, USA email: kgordon@stsci.edu

\begin{abstract}
Early results from the SAGE-SMC (Surveying the Agents of Galaxy Evolution in the tidally-disrupted, low-metallicity Small Magellanic Cloud) Spitzer legacy program are presented. These early results concentrate on the SAGE-SMC MIPS observations of the SMC Tail region. This region is the high $\mathrm{H}$ I column density portion of the Magellanic Bridge adjacent to the SMC Wing. We detect infrared dust emission and measure the gas-to-dust ratio in the SMC Tail and find it similar to that of the SMC Body. In addition, we find two embedded cluster regions that are resolved into multiple sources at all MIPS wavelengths.
\end{abstract}

Keywords. galaxies: individual (SMC), galaxies: ISM, Magellanic Clouds, infrared: ISM

\section{Introduction}

SAGE-SMC is a Spitzer legacy program (cycle 4, 285 hours) to map the entire SMC (Bar, Wing, and Tail) with IRAC and MIPS. The SAGE-SMC observations cover $\sim 30 \mathrm{deg}^{2}$, greatly expanding on the $\mathrm{S}^{3} \mathrm{MC}$ pathfinder survey (Bolatto et al. 2007) which covered the inner $\sim 3 \mathrm{deg}^{2}$ of the SMC. The main SAGE-SMC goal is to study the evolution of a single galaxy in detail. As the SMC is close $(d \sim 60 \mathrm{kpc})$, we can investigate the cycle of star formation and dust by studying the injection of material into the interstellar medium (ISM) from evolved stars, the contents of the present day ISM, and how the ISM is consumed in regions of star formation. The SMC is a unique target for such studies as it is nearby, low metallicity $\left(1 / 5 \mathrm{Z}_{\odot}\right)$, and tidally disrupted. The comparison of the SAGE-SMC observations with similar observations of the LMC (SAGE-LMC, Meixner et al. 2006) and the Milky Way (e.g., GLIMPSE \& MIPSGAL) will provide a solid understanding of galaxy evolution over a wide range of metallicities and star formation histories.

\section{Status of observations}

The SAGE-SMC observations are taken at 2 epochs with instrumental field-of-views rotated by $\sim 90^{\circ}$ to help suppress residual instrumental signatures in both MIPS and 


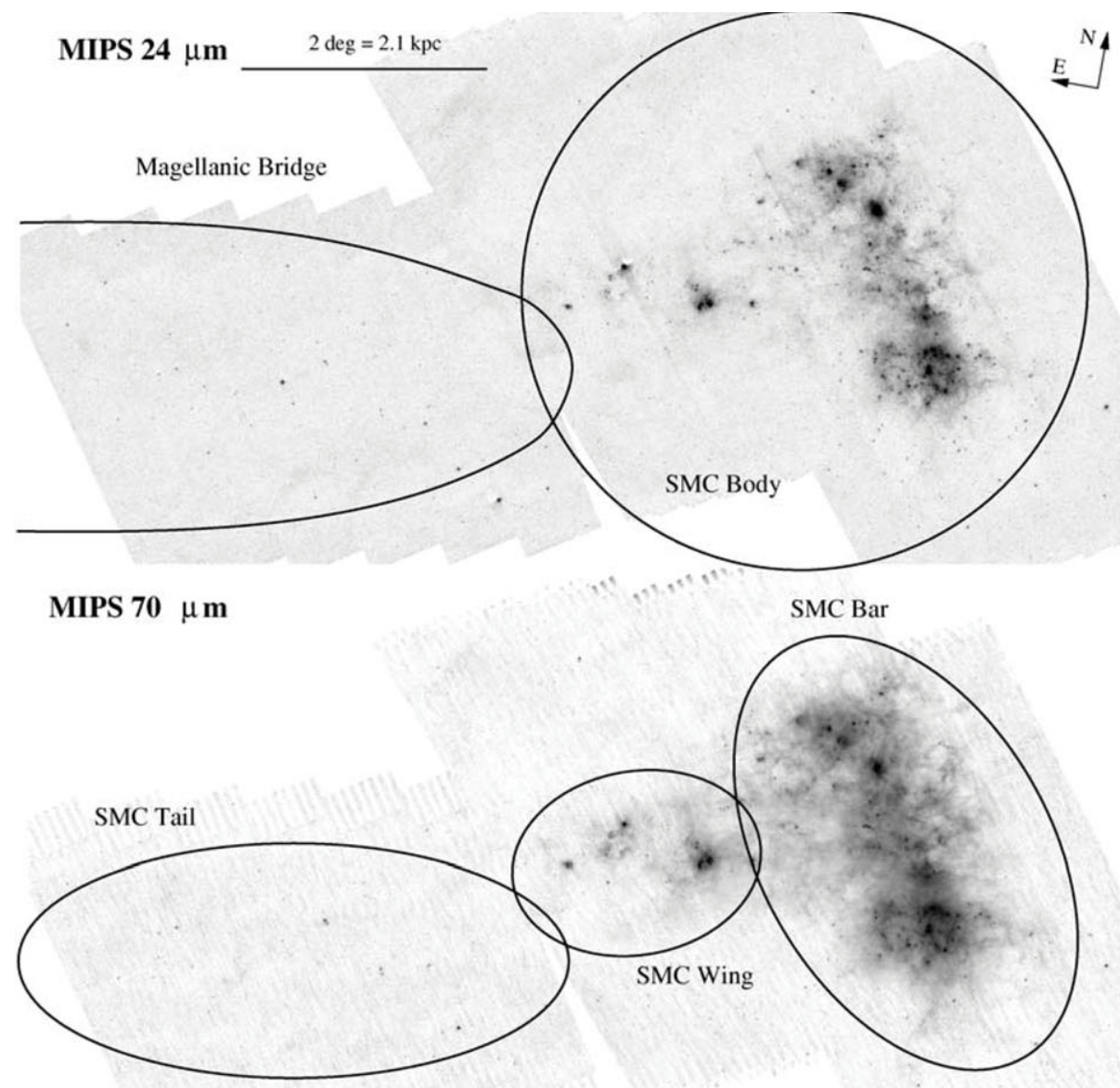

MIPS $160 \mu \mathrm{m}$

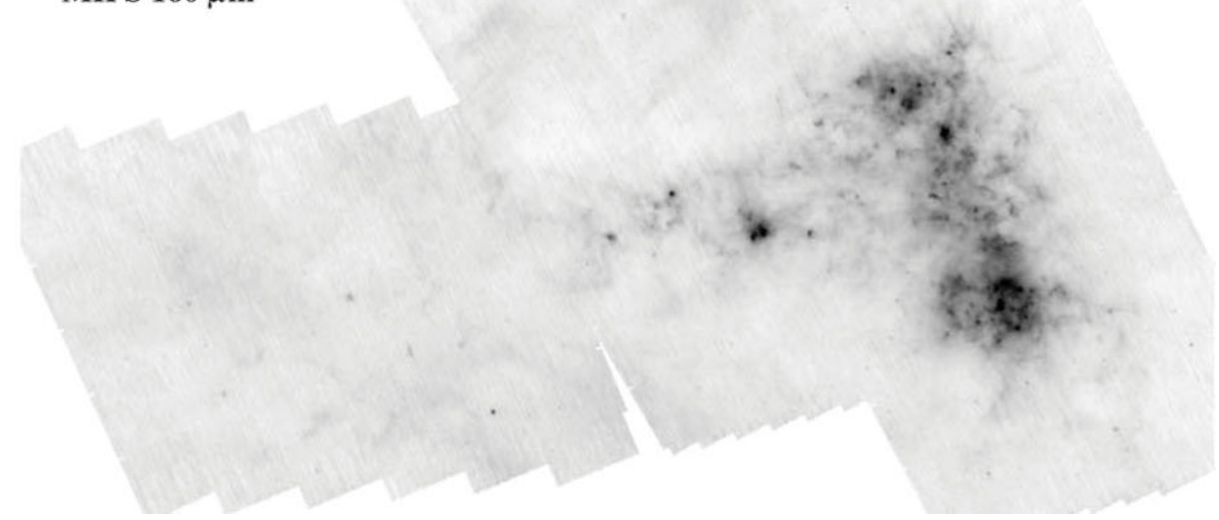

Figure 1. The MIPS 24, 70, and $160 \mu \mathrm{m}$ mosaics of the SMC are shown.

IRAC and allow for studies of variable sources. The observation dates and status of each epoch of observations are given in Table 2. The MIPS $1^{\text {st }}$ epoch observations are the subject of this paper and are displayed in Fig. 1. The $S^{3}$ MC MIPS $70 \& 160 \mu \mathrm{m}$ data have been added to the SAGE-SMC observations to suppress the residual instrumental 


\begin{tabular}{lcl}
\hline Name & Observation Date & Status \\
\hline MIPS $1^{\text {st }}$ epoch & Sep 2007 & Reduced \& Analysis started \\
MIPS 2 $2^{\text {nd }}$ epoch & Jun 2008 & Reduced \\
IRAC $1^{\text {st }}$ epoch & Jun 2008 & Reduced \\
IRAC $2^{\text {nd }}$ epoch & Sep 2008 & Reductions Started \\
\hline
\end{tabular}

signatures in the region of overlap (most of the SMC body and wing). Note that residual baseline drifts in the $\mathrm{S}^{3} \mathrm{MC} 70 \mu \mathrm{m}$ data were subtracted by comparison with the SAGESMC $70 \mu \mathrm{m}$ data before mosaicking. The epoch 1 MIPS $24 \mu \mathrm{m}$ point source catalog includes 13,974 high reliability $(>5 \sigma)$ sources. The similar catalog for the LMC (from SAGE-LMC) includes 39,019 sources.

\section{SMC tail dust}
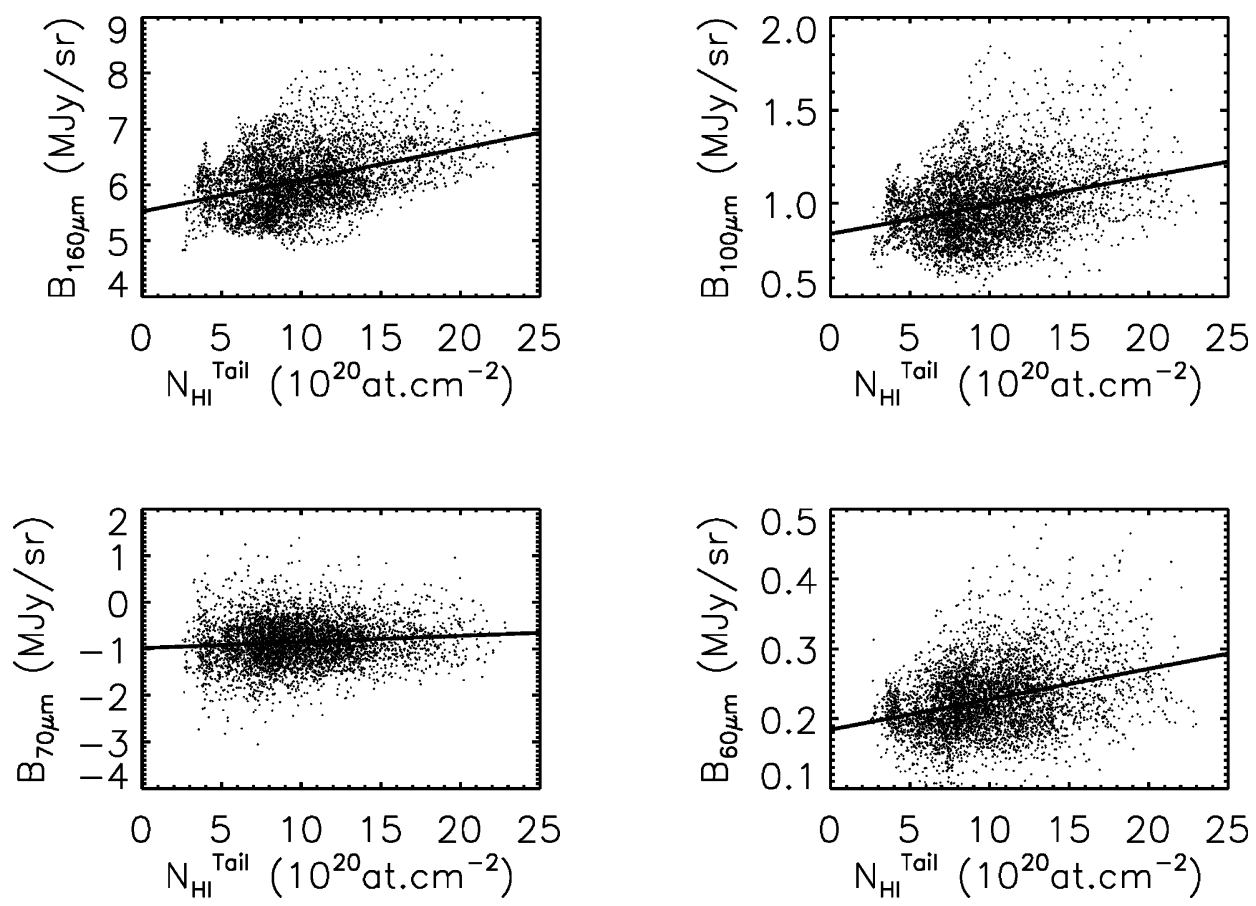

Figure 2. The correlations between the SMC H I column densities and MW foreground subtracted IRAS 25, IRAS 60, MIPS 70, and MIPS $160 \mu \mathrm{m}$ surface brightnesses are shown. The negative surface brightnesses at MIPS $70 \mu \mathrm{m}$ are a result of the subtraction of the time varying instrumental baseline that has clearly resulted in an oversubtraction. This does not affect our measurement of the correlation as we are only interested in the slope.

The Magellanic Bridge connects the SMC and LMC with a bridge of Hi. Directly adjacent to the SMC, there is a high H I density portion which may be related to the SMC. This region likely represents the closest example of tidally stripped material with recent star formation and no old stars (Harris 2007). One of the goals of the SAGESMC observations is to measure the dust content of this region, which we are calling the SMC Tail. The MW cirrus foreground was removed using predictions based on MW $\mathrm{H}$ I foreground measurements. The residual IR emission was correlated with the SMC 
$\mathrm{HI}$ and shown in Fig. 2. Preliminary calculations give an atomic gas-to-dust ratio of $\sim 1000$. This is similar to the SMC Body gas-to-dust ratio (Bot et al. 2004) and indicates that the SMC Tail material has been recently stripped from the SMC Body. This agrees with the measured metallicities of stars in the SMC Tail (Lee et al. 2005) and numerical simulations (Connors et al. 2006).

\section{SMC tail young, embedded clusters}

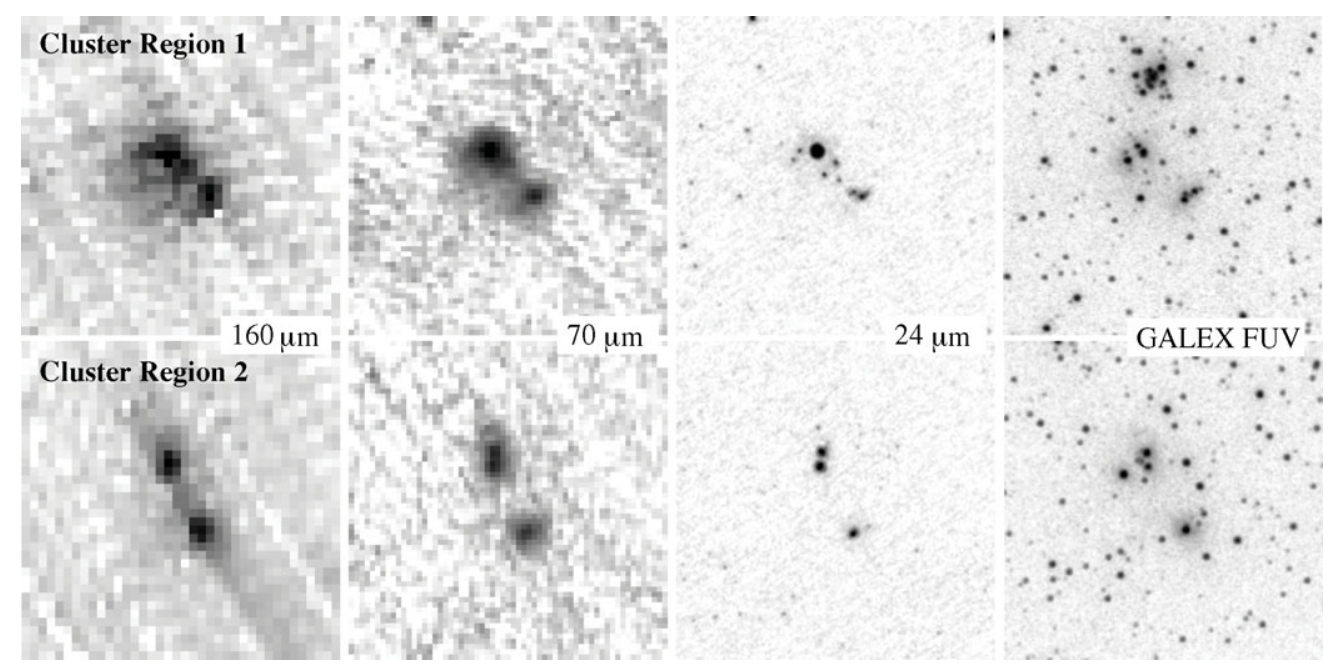

Figure 3. The two cluster regions with resolved sources at all MIPS wavelengths are shown along with the same region observed in the GALEX far-UV band.

The SAGE-SMC observations show a number of point sources in the SMC Tail detected at $24,70, \& 160 \mu \mathrm{m}$. Two are resolved into multiple sources in all MIPS bands and are associated with young, UV bright clusters of stars. These clusters are prime examples of tidally triggered star formation regions which are still embedded in the natal clouds as seen from the H I images. They provide localized measurements of the atomic gas-todust ratio of $\sim 200$. When combined with existing CO observations, the total gas-to-dust ratios are $\sim 250$ to 450 . The 2 nd epoch of MIPS observations will enhance the detections, especially at $160 \mu \mathrm{m}$.

\section{References}

Bolatto, A. D., Simon, J. D., Stanimirović, S., et al. 2007, ApJ, 655, 212

Bot, C., Boulanger, F., Lagache, G., Cambrésy, L., \& Egret, D. 2004, A\& $A, 423,567$

Connors, T. W., Kawata, D., \& Gibson, B. K. 2006, MNRAS, 371, 108

Harris, J. 2007, ApJ, 658, 345

Lee, J.-K., Rolleston, W. R. J., Dufton, P. L., \& Ryans, R. S. I. 2005, A\&A, 429, 1025

Meixner, M., Gordon, K. D., Indebetouw, R., et al. 2006, AJ, 132, 2268 


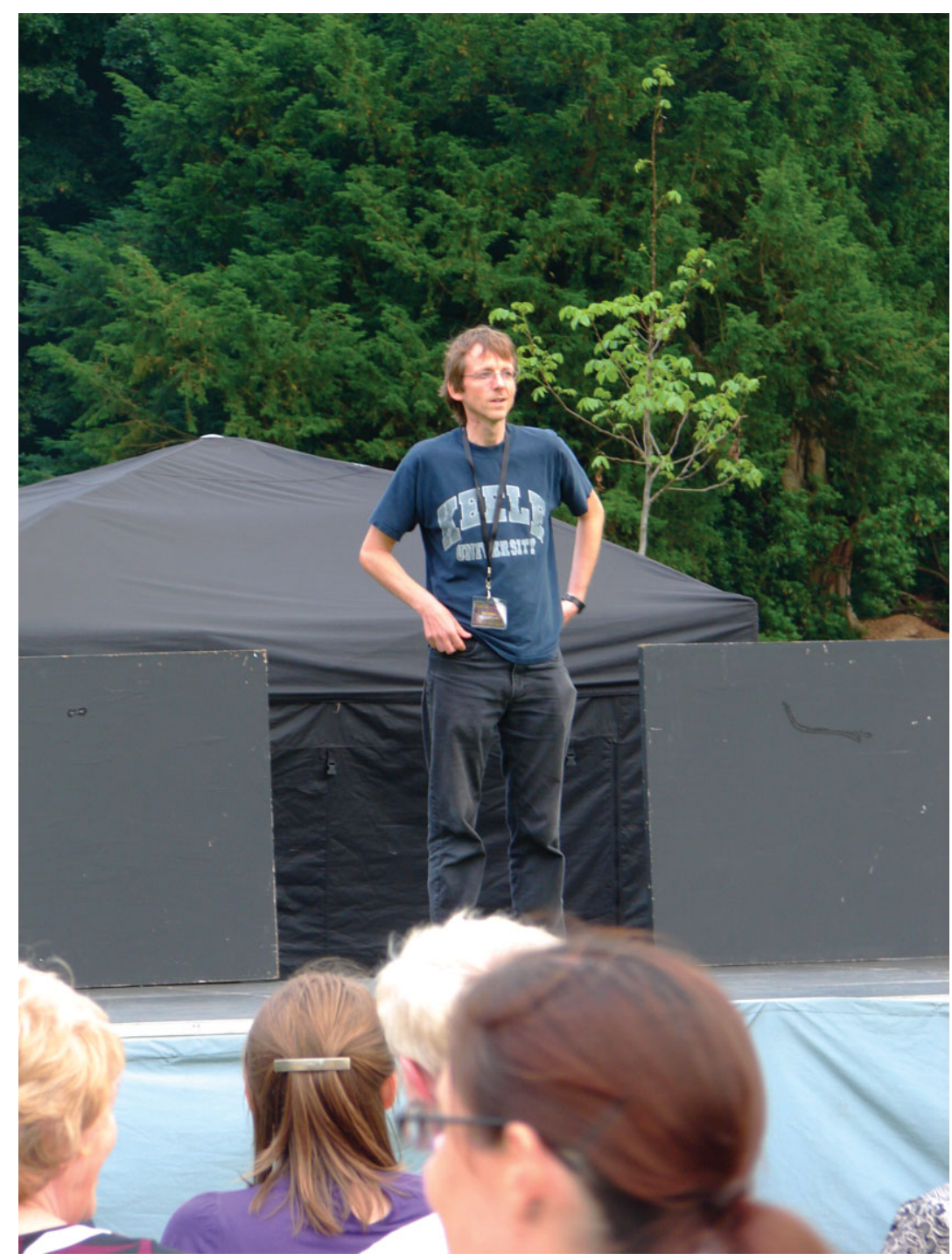

Rob Jeffries, live on stage. Before the drama of Othello, thunder, lighting and rain. 